\title{
Prediction of Amyloid Positivity in Mild Cognitive Impairment Using Fully Automated Brain Segmentation Software
}

This article was published in the following Dove Press journal:

Neuropsychiatric Disease and Treatment

\begin{abstract}
Koung Mi Kang, (iD)' Chul-Ho Sohn, (ID) ${ }^{2}$ Min Soo Byun, (ID ${ }^{3}$ Jun Ho Lee, ${ }^{4}$ Dahyun $Y i$, iD ${ }^{3}$ Younghwa Lee, ${ }^{4}$ Jun-Young Lee, (iD ${ }^{5}$ Yu Kyeong Kim, ${ }^{6}$ Bo Kyung Sohn, (iD) ${ }^{7}$ Roh-Eul Yoo, (iD) ${ }^{1}$ Tae Jin Yun, (DD' Seung Hong Choi, ${ }^{2}$ Jihoon Kim, (D) Dong Young Lee (DD ${ }^{8}$

On behalf of the KBASE Research Group

'Department of Radiology, Seoul National University Hospital, Seoul, Republic of Korea; ${ }^{2}$ Department of Radiology, Seoul National University Hospital, Seoul National University College of Medicine, Seoul, Republic of Korea; ${ }^{3}$ Medical Research Center Seoul National University, Institute of Human Behavioral Medicine, Seoul, Republic of Korea; ${ }^{4}$ Department of Neuropsychiatry, Seoul National University Hospital, Seoul, Republic of Korea; ${ }^{5}$ Department of Neuropsychiatry, SMG-SNU Boramae Medical Center, Seoul National University College of Medicine, Seoul, Republic of Korea; ${ }^{6}$ Department of Nuclear Medicine, SMG-SNU Boramae Medical Center, Seoul National University College of Medicine, Seoul, Republic of Korea; ${ }^{7}$ Department of Psychiatry, Sanggye Paik Hospital, Inje University College of Medicine, Seoul, Republic of Korea; ${ }^{8}$ Department of Neuropsychiatry, Seoul National University Hospital, Seoul National University

College of Medicine, Seoul, Republic of Korea
\end{abstract}

Correspondence: Chul-Ho Sohn

Department of Radiology, Seoul National

University Hospital, Seoul National University

College of Medicine, I0I Daehak-Ro, Jongno-

$\mathrm{Gu}$, Seoul 03080, Republic of Korea

Tel +82-2-207203972

Fax +82-2-747-7418

Email neurorad63@gmail.com

Dong Young Lee

Department of Neuropsychiatry, Seoul National University Hospital , Seoul National University College of Medicine, I0I Daehak-Ro, Jongno-

$\mathrm{Gu}$, Seoul 03080, Republic of Korea

Tel +82-2-2072-2205

Fax +82-2-744-724l

Email selfpsy@snu.ac.kr
Objective: To assess the predictive ability of regional volume information provided by fully automated brain segmentation software for cerebral amyloid positivity in mild cognitive impairment (MCI).

Methods: This study included 130 subjects with amnestic MCI who participated in the Korean brain aging study of early diagnosis and prediction of Alzheimer's disease, an ongoing prospective cohort. All participants underwent comprehensive clinical assessment as well as ${ }^{11} \mathrm{C}$-labeled Pittsburgh compound PET/MRI scans. The predictive ability of volumetric results provided by automated brain segmentation software was evaluated using binary logistic regression and receiver operating characteristic curve analysis.

Results: Subjects were divided into two groups: one with $A \beta$ deposition (58 subjects) and one without $A \beta$ deposition (72 subjects). Among the varied volumetric information provided, the hippocampal volume percentage of intracranial volume $(\% \mathrm{HC} / \mathrm{ICV})$, normative percentiles of hippocampal volume $\left(\mathrm{HC}_{\text {norm }}\right)$, and gray matter volume were associated with amyloid- $\beta$ (A $\left.\beta\right)$ positivity (all $P<0.01$ ). Multivariate analyses revealed that both $\% \mathrm{HC} / \mathrm{ICV}$ and $\mathrm{HC}_{\text {norm }}$ were independent significant predictors of $\mathrm{A} \beta$ positivity (all $P<0.001$ ). In addition, prediction scores derived from $\% \mathrm{HC} / \mathrm{ICV}$ with age and $\mathrm{HC}_{\text {norm }}$ showed moderate accuracy in predicting $\mathrm{A} \beta$ positivity in MCI subjects (the areas under the curve: 0.739 and 0.723 , respectively).

Conclusion: Relative hippocampal volume measures provided by automated brain segmentation software can be useful for screening cerebral $A \beta$ positivity in clinical practice for patients with amnestic MCI. The information may also help clinicians interpret structural MRI to predict outcomes and determine early intervention for delaying the progression to Alzheimer's disease dementia.

Keywords: amyloid, brain segmentation, magnetic resonance imaging, cognitive impairment

\section{Introduction}

Mild cognitive impairment (MCI) is an intermediate state between normal and Alzheimer's disease (AD), ${ }^{1}$ and amnestic MCI has been considered a prodromal stage of $\mathrm{AD}$ dementia. ${ }^{2}$ However, approximately $40 \%$ of amnestic MCI individuals do not show sufficient cerebral amyloid- $\beta$ (A $\beta)$ deposition for the diagnosis of AD. ${ }^{3-6}$ Among amnestic MCI subjects, amyloid-positive $(A \beta+)$ subjects were much more likely to develop dementia than were amyloid-negative (A $\beta$-) subjects (50\% vs $19 \%$ within 2 years). ${ }^{7}$ Therefore, for early therapeutic intervention, the detection of high levels of $\mathrm{A} \beta$ deposition in MCI subjects is invaluable. 
At present, the most well developed in vivo measures of cerebral $A \beta$ deposition are cerebrospinal fluid (CSF) analyses and positron emission tomography (PET) ${ }^{8}$ However, CSF biomarkers and PET imaging are neither affordable nor widely available in all clinical settings. Therefore, since availability varies across countries, research towards more accessible diagnostic tools for an early AD diagnosis is still needed. ${ }^{9-11}$

Conversely, structural brain MRI is a completely noninvasive and widely used method to exclude other structural abnormalities and diagnose neurodegeneration in subjects with suspected $\mathrm{AD} .{ }^{12}$ In particular, the association between the regional brain atrophy rate and $A \beta$ load has been reported in MCI. ${ }^{13}$ Currently, there are several available software packages for brain segmentation and morphometry: FSL, ${ }^{14}$ FreeSurfer, ${ }^{15}$ and Statistical Parametric Mapping. ${ }^{16}$ However, the use of these software programs in routine clinical practice is limited due to their laborintensive nature.

Recently, a US Food and Drug Administrationapproved fully automated brain segmentation software (NeuroQuant, CorTechs Laboratories) has been introduced for clinical use. ${ }^{16}$ This tool provides absolute and relative volumes of various brain structures and normative percentiles compared with age-appropriate references in 6-8 minutes. ${ }^{17}$ Several previous studies reported the clinical usefulness of NeuroQuant to aid AD diagnosis or compare MCI converters vs nonconverters. ${ }^{17-19}$

Therefore, our aim was to assess the predictive ability of regional volume information provided by NeuroQuant for cerebral $A \beta$ positivity in amnestic MCI individuals.

\section{Materials and Methods}

\section{Participants}

This study is based on the Korean Brain Aging Study for Early Diagnosis and Prediction of Alzheimer's Disease (KBASE), ${ }^{20}$ an ongoing, prospective, community-based cohort study. As of February 2017, 130 amnestic MCI subjects were included. MCI diagnosis was established according to the recommendations of the National Institute on Aging-Alzheimer's Association (NIA-AA) guidelines. $^{21}$ The inclusion criteria for amnestic MCI were as follows: (a) memory complaints corroborated by the patient, an informant, or clinician; (b) objective memory impairment for age, education, and sex (ie, at least 1.0 standard deviation below the respective age, education, and sex-specific mean for at least one of the four episodic memory tests included in the Korean version of the Consortium to Establish a Registry for Alzheimer's Disease [CERAD-K] neuropsychological battery [word list memory, word list recall, word list recognition and constructional recall test]); (c) largely intact functional activities; and (d) no dementia. The global clinical dementia rating (CDR) score of all MCI individuals was 0.5 . The exclusion criteria were as follows: (a) presence of major psychiatric illness; (b) significant neurological or medical condition or comorbidities that could affect mental function; (c) contraindications to MRI (eg, pacemaker, claustrophobia); (d) illiteracy; (e) presence of significant visual/ hearing difficulty; severe communication or behavioral problems that would make a clinical examination or a brain scan difficult; (f) use of an investigational drug; and $(\mathrm{g})$ women who were pregnant or breastfeeding. More detailed information on recruitment of the KBASE cohort was described in our previous report. ${ }^{20}$ This study protocol was approved by the Institutional Review Boards of Seoul National University Hospital and Seoul National University-Seoul Metropolitan Government Boramae Center, and all subjects provided written informed consent.

\section{Clinical Assessment}

All subjects were examined by trained psychiatrists who had advanced training in dementia research using the CERAD-K based on the Korean clinical assessment protocol. $^{20}$

\section{Image Acquisition}

All subjects underwent simultaneous three-dimensional (3D) $\left[{ }^{11} \mathrm{C}\right]$ Pittsburgh compound B (PiB)-PET and 3D T1weighted magnetization-prepared rapid acquisition with gradient echo (MPRAGE) sequence MRI scans using a 3.0T Biograph mMR (PET-MR) scanner (Siemens, Washington DC, USA) according to the manufacturer's approved guidelines. 3D T1-weighted images were acquired in the sagittal orientation, and the acquisition parameters were as follows: repetition time $=1670 \mathrm{~ms}$, echo time $=1.89 \mathrm{~ms}$, field of view $=250 \mathrm{~mm}$, and a $256 \times$ 256 matrix with a $1.0-\mathrm{mm}$ slice thickness. For PiB-PET, a 30-minute emission scan was obtained 40 minutes after intravenous administration of $555 \mathrm{MBq}$ of $\left[{ }^{11} \mathrm{C}\right] \mathrm{PiB}$ (range, 450-610 MBq). The PiB-PET data collected in list mode were processed for routine corrections such as uniformity, UTE-based attenuation, and decay corrections and were reconstructed into a $344 \times 344$ image matrix using iterative methods (5 iterations with 21 subsets). 


\section{Volumetric MRI Analyses}

For each subject, a set of T1-weighted MPRAGE images was processed by the NeuroQuant software package (CorTechs Labs) and FreeSurfer software (version 5.3, http://surfer.nmr. mgh.harvard.edu/). NeuroQuant performed the following steps: correction for gradient nonlinearity and B1 field inhomogeneity before skull stripping, removal of the scalp, skull, and meninges; inflation of the brain to a spherical shape; mapping of the spherical brain to a common spherical space shared with the Talairach atlas coordinates; identification of segmented brain regions; and deflation of the brain to its original shape while retaining the identifying information for the segmented brain regions. ${ }^{22}$ Each brain region volume was corrected for interindividual differences in head size by dividing by the intracranial volume (ICV), and the results are expressed as a volume percentage (\%) of $\mathrm{ICV}^{22}$ A normative percentile value for each volume $\%$ of ICV was also provided based on the data of healthy control subjects provided by the software. As a result, the report for each subject included absolute volume, volume \% of ICV and normative percentile value of the whole brain, lateral ventricles, thalami, cortical gray matter (GM), cerebral white matter (WM), WM hypointensities, third ventricle, hippocampi and inferior lateral ventricles.

The same T1-weighted MR images were also segmented using the recon-all script of FreeSurfer version 5.3 on each T1weighted scan. ${ }^{15}$ FreeSurfer segmentation outputs were each visually inspected by trained image analysts for moderate to severe errors. When errors occurred, trained image analysts performed manual correction of FreeSurfer segmentations. Volumes in each region of interest (ROI) of FreeSurfer's atlas were obtained from FreeSurfer's output aseg.stats files. For the evaluation of intermethod reliability, the volumes of various brain regions from the NeuroQuant General Morphometry Report were compared with those from FreeSurfer.

\section{Measurement of $A \beta$ Deposition}

The mean regional ${ }^{11} \mathrm{C}-\mathrm{PiB}$ uptake values from the frontal, posterior cingulate-precuneus, lateral temporal and lateral parietal regions were extracted using the individual AAL116 atlas from T1-coregistered PiB-PET images. ${ }^{23,24}$ The standardized uptake value ratios (SUVRs) for each ROI were calculated by dividing the mean value for all voxels within each ROI by the mean cerebellar uptake value due to its relatively low $\mathrm{A} \beta$ deposition. ${ }^{25}$ Each subject was classified as $A \beta$ positive if the SUVR was $>1.4$ in at least one of the four ROIs or as $A \beta$ negative if the SUVR of all four
ROIs was $\leq 1.4 .{ }^{26}$ Details of $\left[{ }^{11} \mathrm{C}\right]$ PiB-PET image preprocessing are described in Supplementary Methods S1.

\section{Statistical Analysis}

Differences between $\mathrm{A} \beta+$ and $\mathrm{A} \beta$ - MCI subjects for demographic and clinical characteristics were described using independent $t$-tests or chi-square tests. Intermethod reliabilities were measured by calculating interclass correlation coefficients (ICC) using a 2-way mixed model with consistency type. ${ }^{27}$ ICC values of $0-0.40,0.40-0.75$, or greater than 0.75 indicated poor, fair to good, or excellent reliability. ${ }^{27}$

To investigate whether a relative regional volume measure (ie, volume $\%$ of ICV or its normative percentile value) was associated with $A \beta$ positivity in MCI subjects, two steps of analysis were independently conducted for volume $\%$ of ICV and normative percentile value. In the first step, a binary logistic regression with the enter method was applied to each brain region. For the volume \% of ICV, age and sex were adjusted for correction, while no covariate was included in the model for normative percentile value. Variance influence factors were calculated to assess multicollinearity between multiple independent variables. Brain regions with $P<0.1$ in the first step were selected for the second step. In the second step, multivariate backward logistic regression models were used to determine the independent predictors of $\mathrm{A} \beta$ positivity. Receiver operating characteristic (ROC) curve analysis was also performed for significant predictor(s) to evaluate the prediction accuracy. If there were multiple predictors, a prediction score was calculated as the sum of each predictor multiplied by the respective $\beta$ coefficients from the final logistic regression model. As the optimal cutoff point for predicting $A \beta$ positivity can vary according to the purpose, ie, screening candidates for clinical trials or screening patients who need further evaluation using amyloid PET, we investigated how the sensitivity, specificity, positive predictive value, negative predictive value, and accuracy value change as the relative regional volume measure or prediction score changes instead of determining a single optimal cutoff value. Statistical analysis was performed with commercially available software (SPSS, version 23.0 for Windows; and MedCalc, version 9.3.0.0).

\section{Results}

Data on the demographic and clinical characteristics of MCI subjects are presented in Table 1. The mean age of MCI subjects was $73.4 \pm 7.0$ years (range, $57-90$ years), and $66 \%$ of the subjects were female. There was no significant difference in age, sex or educational level between the $\mathrm{A} \beta+$ and $\mathrm{A} \beta-\mathrm{MCI}$ subjects. 
Table I Demographic and Clinical Characteristics of MCI Subjects

\begin{tabular}{|l|l|l|l|l|}
\hline Variables & All & A $\boldsymbol{\beta}$ - $(\mathbf{n}=\mathbf{7 2})$ & A $\boldsymbol{\beta}+\mathbf{( n = 5 8 )}$ & P-value \\
\hline Age (y) & & & & 0.900 \\
$\quad$ Mean \pm SD & $73.4 \pm 7.0$ & $73.5 \pm 7.4$ & $73.3 \pm 6.5$ & \\
Range & $57-90$ & $58-90$ & $57-88$ & 0.814 \\
\hline Sex (n) & & & 19 & \\
Men & 44 & 25 & 39 & \\
Women & 86 & 47 & $10.5 \pm 4.3$ & 0.289 \\
\hline Education (y, mean \pm SD) & $10.1 \pm 4.5$ & $9.7 \pm 4.7$ & $21.8 \pm 3.1$ & 0.003 \\
MMSE & $22.6 \pm 3.1$ & $23.4 \pm 2.9$ & \\
\hline
\end{tabular}

Note: Demographic and clinical characteristics were compared using independent t-tests or chi-square tests.

Abbreviations: $\mathrm{MCl}$, mild cognitive impairment; $\mathrm{A} \beta$, beta-amyloid; $y$, years; SD, standard deviation; n, number; MMSE, Mini-Mental Status Examination.

\section{Intermethod Reliability Between}

\section{NeuroQuant and FreeSurfer}

The intermethod reliability between NeuroQuant and FreeSurfer was excellent in the 14 supratentorial brain regions (ICC $=0.790-0.994$ ) except for the pallidum $(\mathrm{ICC}=-0.015$, Table 2). Absolute volume measures are shown in Supplementary Table 1.

\section{Association of Regional Volume Measures Reported by NeuroQuant with A $\beta$ Positivity} There was no significant multicollinearity between the independent variables (variance inflation factor $=1.254$ 3.839 for the mean volume \% of ICV and 1.037-2.635 for the normative percentile, Supplementary Table 2). The

Table 2 Intermethod Reliability Between NeuroQuant and FreeSurfer

\begin{tabular}{|l|l|l|}
\hline & ICC & $\mathbf{9 5 \%} \mathbf{~ C I}$ \\
\hline Total intracranial volume & 0.981 & $0.973,0.986$ \\
Whole-brain parenchyma & 0.975 & $0.965,0.982$ \\
Forebrain parenchyma & 0.982 & $0.975,0.987$ \\
Cortical GM & 0.935 & $0.908,0.954$ \\
Cerebral WM & 0.913 & $0.876,0.938$ \\
Lateral ventricle & 0.994 & $0.992,0.996$ \\
Inferior lateral ventricle & 0.981 & $0.973,0.986$ \\
3rd ventricle & 0.988 & $0.983,0.991$ \\
Caudate & 0.790 & $0.703,0.852$ \\
Putamen & 0.808 & $0.729,0.865$ \\
Pallidum & -0.015 & $-0.436,0.282$ \\
Thalamus & 0.894 & $0.850,0.925$ \\
Amygdala & 0.904 & $0.864,0.932$ \\
Hippocampus & 0.960 & $0.944,0.972$ \\
WM hypointensities & 0.933 & $0.905,0.952$ \\
\hline
\end{tabular}

Note: ICC, interclass correlation coefficient; $\mathrm{Cl}$, confidence interval; GM, gray matter; WM, white matter. mean volume \% of ICV and normative percentile of the cortical GM and hippocampus were significantly larger in $A \beta$ - than in $A \beta+$ MCI subjects (Table 3). The first step of binary logistic regression for volume \% of ICV indicated that among various brain regions, the cerebral GM and hippocampus were significantly associated with $A \beta$ positivity after adjusting for age and sex (Table 3). The second-step multivariate logistic regression revealed that age and hippocampal volume $\%$ of ICV $(\% \mathrm{HC} / \mathrm{ICV})$ were independently associated with $\mathrm{A} \beta$ positivity (odds ratio, $0.917 ; 95 \% \mathrm{CI}, 0.858-0.980, P=0.011$ for age; odds ratio per unit of $0.001 \%, 0.986$; $95 \%$ CI, 0.979 $0.992, P<0.001$ for $\% \mathrm{HC} / \mathrm{ICV})$. A prediction score $\left(\mathrm{HC}_{\text {age }}\right)$ was calculated using age and $\% \mathrm{HC} / \mathrm{ICV}$ with the respective $\beta$ coefficients from the logistic regression models $\left(\mathrm{Y}=12.047+-0.087 \mathrm{X}_{1}+-14.192 \mathrm{X}_{2}\right.$, where $\mathrm{Y}$ is the prediction score of $\mathrm{HC}_{\text {age }}, \mathrm{X}_{1}$ is age, and $\mathrm{X}_{2}$ is $\% \mathrm{HC} / \mathrm{ICV}$ ).

Regarding the normative percentile value of each regional volume, the cortical GM, inferior lateral ventricle, and hippocampal values were related to $A \beta$ positivity in the first-step binary logistic regression analyses (Table 3 ). The second-step multivariate logistic regression analysis showed that only the normative percentile of hippocampal volume $\left(\mathrm{HC}_{\text {norm }}\right)$ was independently associated with $\mathrm{A} \beta$ positivity (odds ratio, 0.977; 95\% CI, 0.966-0.988, $P<0.001)$.

Structural MRI and PiB-PET images for representative cases of $A \beta$ - and $A \beta+$ MCI subjects are shown in Figures 1 and 2.

\section{Performance of Hippocampal Volume Measures for $A \beta$ Positivity Prediction in $\mathrm{MCl}$}

In the ROC curve analysis, the areas under the curve (AUCs) for $\mathrm{A} \beta$ positivity prediction of $\mathrm{HC}_{\mathrm{age}}$ and 
Table 3 Relative Regional Volumes as a Percentage of ICV and Normative Percentile in A $\beta$ - and A $\beta+M C I$ Subjects

\begin{tabular}{|c|c|c|c|c|c|}
\hline & $A \beta-(n=72)$ & $A \beta+(n=58)$ & P-value & Odds Ratio & $95 \% \mathrm{Cl}$ for Odds Ratio \\
\hline \multicolumn{6}{|l|}{ Percentage (\%) of ICV } \\
\hline Whole-brain parenchyma & $74.82 \pm 3.47$ & $74.23 \pm 3.59$ & 0.222 & 0.923 & $0.812,1.049$ \\
\hline Cortical GM & $29.31 \pm 2.58$ & $28.04 \pm 2.18$ & 0.002 & 0.753 & $0.632,0.898$ \\
\hline Cerebral WM & $32.16 \pm 1.73$ & $32.43 \pm 1.59$ & 0.341 & 1.120 & $0.886,1.416$ \\
\hline Lateral ventricle & $2.77 \pm 1.03$ & $2.88 \pm 0.99$ & 0.886 & 1.163 & $0.798,1.694$ \\
\hline Inferior lateral ventricle & $0.20 \pm 0.09$ & $0.22 \pm 0.09$ & 0.104 & $1.041 \dagger$ & $0.922,1.093 \dagger$ \\
\hline Third ventricle & $0.16 \pm 0.04$ & $0.16 \pm 0.05$ & 0.300 & $1.049 \dagger$ & $0.958,1.149 \dagger$ \\
\hline Thalamus & $1.03 \pm 0.08$ & $1.02 \pm 0.06$ & 0.451 & 0.129 & $0.001,26.771$ \\
\hline Hippocampus & $0.44 \pm 0.08$ & $0.39 \pm 0.07$ & $<0.001$ & $0.985 \ddagger$ & $0.978,0.992 \ddagger$ \\
\hline WM hypointensities & $0.53 \pm 1.60$ & $0.15 \pm 0.30$ & 0.186 & 0.630 & $0.3|7,1.25|$ \\
\hline \multicolumn{6}{|l|}{ Normative percentile } \\
\hline Whole-brain parenchyma & $78.32 \pm 28.56$ & $72.34 \pm 27.20$ & 0.228 & 0.992 & $0.980,1.005$ \\
\hline Cortical GM & $36.78 \pm 29.21$ & $23.19 \pm 24.17$ & 0.007 & $0.98 I$ & $0.968,0.995$ \\
\hline Cerebral WM & $90.83 \pm 11.96$ & $92.03 \pm 11.59$ & 0.563 & 1.009 & $0.979,1.040$ \\
\hline Lateral ventricle & $59.64 \pm 27.49$ & $63.10 \pm 27.22$ & 0.471 & 1.005 & $0.992,1.018$ \\
\hline Inferior lateral ventricle & $71.97 \pm 24.07$ & $79.36 \pm 23.59$ & 0.084 & 1.014 & $0.998,1.029$ \\
\hline Third ventricle & $66.78 \pm 22.47$ & $69.47 \pm 26.36$ & 0.528 & 1.005 & $0.990,1.019$ \\
\hline Thalamus & $91.97 \pm 13.90$ & $91.88 \pm 15.04$ & 0.971 & 1.000 & $0.976,1.024$ \\
\hline Hippocampus & $54.63 \pm 34.85$ & $27.72 \pm 31.32$ & $<0.001$ & 0.977 & $0.966,0.988$ \\
\hline WM hypointensities & $42.53 \pm 34.00$ & $47.76 \pm 33.43$ & 0.378 & 1.005 & $0.994,1.015$ \\
\hline
\end{tabular}

Notes: Data are the means \pm standard deviations. As the values of \% of ICV of the inferior lateral ventricle, third ventricle, and hippocampus were too small, odds ratios and $95 \% \mathrm{Cls}$ were used to predict the probability of an event occurring based on $0.0 \mathrm{I}$ (inferior lateral ventricle and third ventricle) $\dagger$ or $0.00 \mathrm{I}$ (hippocampus) $\ddagger$ unit change when age and sex are kept constant.

Abbreviations: ICV, intracranial volume; $\mathrm{A} \beta$, beta-amyloid; $\mathrm{MCl}$, mild cognitive impairment; $\mathrm{Cl}$, confidence interval; $\mathrm{GM}$, gray matter; WM, white matter.

$\mathrm{HC}_{\text {norm }}$ were $0.739(95 \% \mathrm{CI}: 0.654,0.812)$ and 0.723 (95\% CI: 0.638, 0.798), respectively (Figure 3). There was no significant difference between the two ROC curves $(P=0.554)$. Table 4 shows the sensitivity, specificity, positive predictive value, negative predictive value, and accuracy at various cutoff points of $\mathrm{HC}_{\mathrm{age}}$ and $\mathrm{HC}_{\text {norm }}$.

\section{Discussion}

In this study, we investigated the predictive accuracy of the relative regional volume value provided by a commercially available brain volumetric software package for the presence of high $\mathrm{A} \beta$ deposition in subjects with MCI. We found that $\mathrm{A} \beta$ positivity could be predicted with only relative volume measures of the hippocampus, ie, $\mathrm{HC}_{\mathrm{age}}$ and $\mathrm{HC}_{\text {norm }}$.

The results of automated volumetric brain assessment and its clinical application have been reported previously. ${ }^{17,28-31}$ In particular, previous studies have shown that the application of fully automated quantification of regional brain volumes on structural MRI of subjects at risk for $\mathrm{AD}$ could improve the detection of focal atrophy of $\mathrm{AD}^{17}$ and predict clinical decline. ${ }^{32,33}$ Our study found that easily accessible software could not only provide regional brain volumes but also predict the likelihood of $A \beta$ positivity in subjects with MCI in a reasonably short processing time of 6-8 minutes. As $A \beta+$ MCI subjects were much more likely to progress to dementia than $\mathrm{A} \beta$ - MCI subjects, ${ }^{7,34}$ in vivo detection of high amyloid repositioning in MCI patients with amyloid PET can provide valuable prognostic information. However, its use is limited in routine clinical practice for MCI workup due to its high cost and limited availability. On the other hand, structural MRI is performed far more often than amyloid PET in MCI patients to rule out the second etiology and to find neuronal injury. ${ }^{8}$ Therefore, our results can be helpful in screening MCI due to AD before an amyloid PET scan is conducted or when it is not available.

GM and hippocampal atrophy are highly validated biomarkers of neuronal injury ${ }^{35}$ following amyloid deposition in $\mathrm{AD} .{ }^{36}$ In our study, among the multiple regional volume measures provided by NeuroQuant, both cerebral GM and hippocampal measures were associated with $A \beta$ positivity in the first exploratory step of analysis. In the final multivariate analyses, however, $\mathrm{A} \beta$ positivity was related only to relative volume measures of the hippocampus, ie, $\mathrm{HC}_{\mathrm{age}}$ and $\mathrm{HC}_{\text {norm }}$, but not to $\mathrm{GM}$ volume. These results are probably due to the well-known phenomenon 


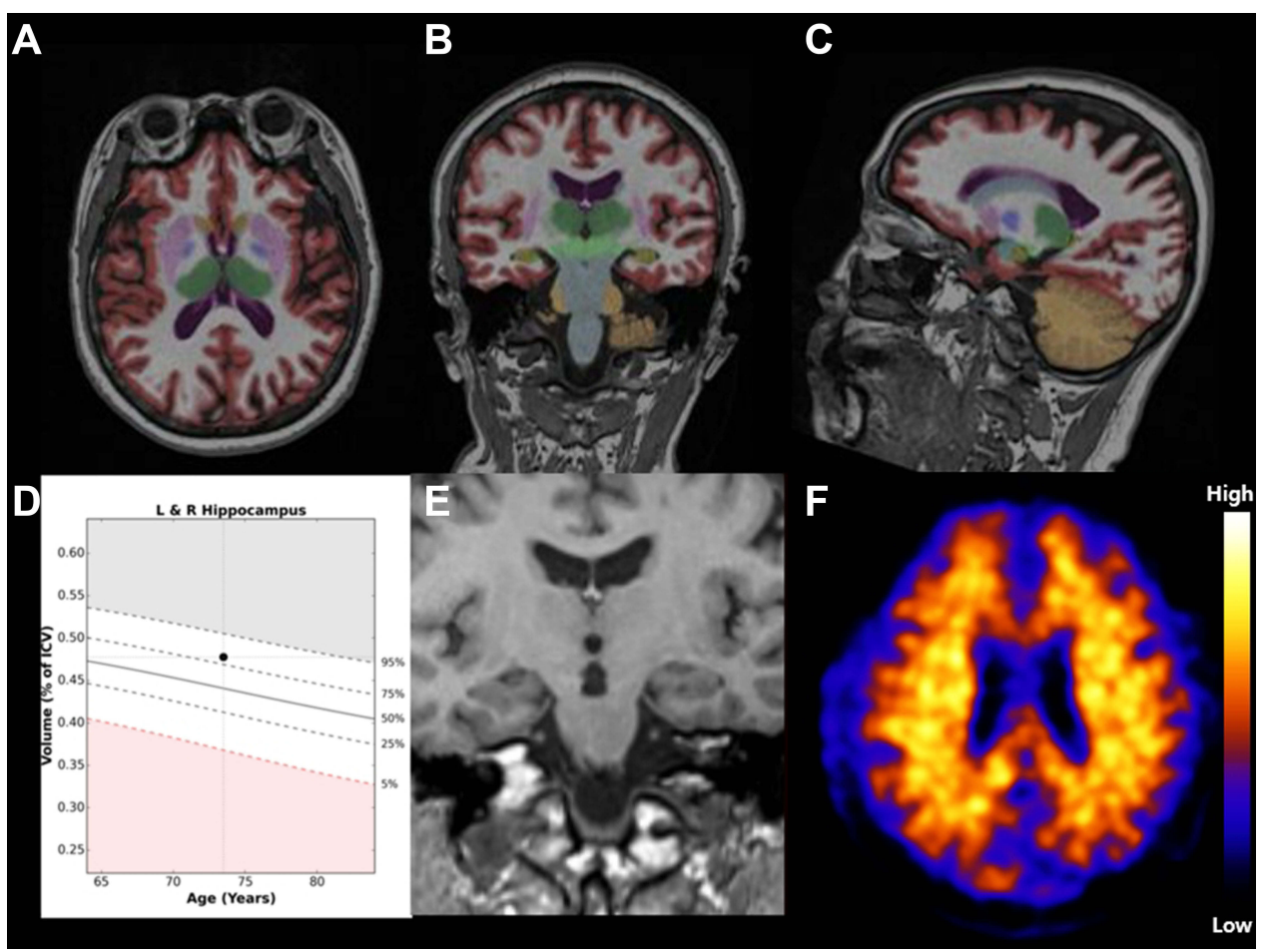

Figure I Representative 73-year-old woman with $\mathrm{MCl}$. (A-C): Output from analysis with NeuroQuant shows segmentation of regional brain volumes.(D): Age-matched reference chart provided by NeuroQuantshowing the \% of ICV and normative percentiles of hippocampal volume are 0.48 and $8 \mathrm{I}$, respectively. The prediction score calculated from age and hippocampal \% of ICV was -13.16. The normative percentile of the hippocampus was 28.(E): Coronal TI-weighted MR image shows no hippocampal atrophy.(F): Negative amyloid imaging scans with "low" Pittsburgh compound B retention.

Abbreviations: $\mathrm{MCl}$, mild cognitive impairment; L, left; R, right; ICV, intracranial volume (Courtesy of CorTechs Laboratories).

that earlier atrophic change in AD occurs in the hippocampus, consistent with early episodic memory deficits followed by later atrophic changes in the temporal, parietal and frontal neocortices. ${ }^{37}$ Hippocampal atrophy is closer in time to amyloid deposition than GM atrophy is.

In contrast to $\mathrm{HC}_{\text {norm }}$, a relative value referring to ageappropriate normative data that are embedded in the NeuroQuant, ${ }^{16} \% \mathrm{HC} / \mathrm{ICV}$ should be adjusted for age because global and regional brain atrophy are part of normal aging. ${ }^{38}$ Therefore, we calculated a prediction score, ie, $\mathrm{HC}_{\text {age }}$, by using a regression equation including both age and $\% \mathrm{HC} / \mathrm{ICV}$. However, as it is cumbersome to calculate the prediction score every time and there was no significant difference in the AUC of ROC curves between $\mathrm{HC}_{\text {age }}$ and $\mathrm{HC}_{\text {norm }}, \mathrm{HC}_{\text {norm }}$ may be more convenient in daily clinical practice.

We evaluated the intermethod reliability between NeuroQuant and FreeSurfer, a widely used volumetric software with research purposes. The ICC was excellent in the 14 supratentorial brain regions $(\mathrm{ICC}=0.790-0.994)$ except the pallidum (ICC $=-0.015$ ). Our results were similar to those of a previous study by Ochs et $\mathrm{al},{ }^{27}$ which demonstrated that NeuroQuant and FreeSurfer showed good to excellent intermethod reliability in volumetric measurements for all brain regions examined (ICC: 0.62 to 0.99 ), with the only exceptions being the pallidum (ICC: 0.029) and cerebellar WM (ICC: 0.031). As previously described, the poor reliability for NeuroQuant versus FreeSurfer measurements of the pallidum is probably due to the similar intensities of the pallidum and WM on T1-weighted MRI. ${ }^{34}$

Some limitations need to be mentioned. First, the prediction of $\mathrm{HC}_{\text {age }}$ and $\mathrm{HC}_{\text {norm }}$ for $\mathrm{A} \beta$ positivity in MCI was not highly accurate given that the AUC was $0.723 \sim 0.739$. This may be an inevitable limitation considering the temporal ordering of $\mathrm{AD}$ pathological changes. That is, $A \beta$ deposition begins at the preclinical stage and almost saturates in the cognitively impaired stages of $\mathrm{AD}$, including $\mathrm{MCI}$, while regional neurodegeneration (including hippocampal atrophy) gradually progresses from MCI to AD dementia. ${ }^{39}$ Second, although we excluded patients with prominent neurological or medical comorbidities, biological factors with the potential to confound the assessment of brain volume changes, such as cardiovascular risk factors, level of hydration, diurnal variations, and alcohol/caffeine use, 


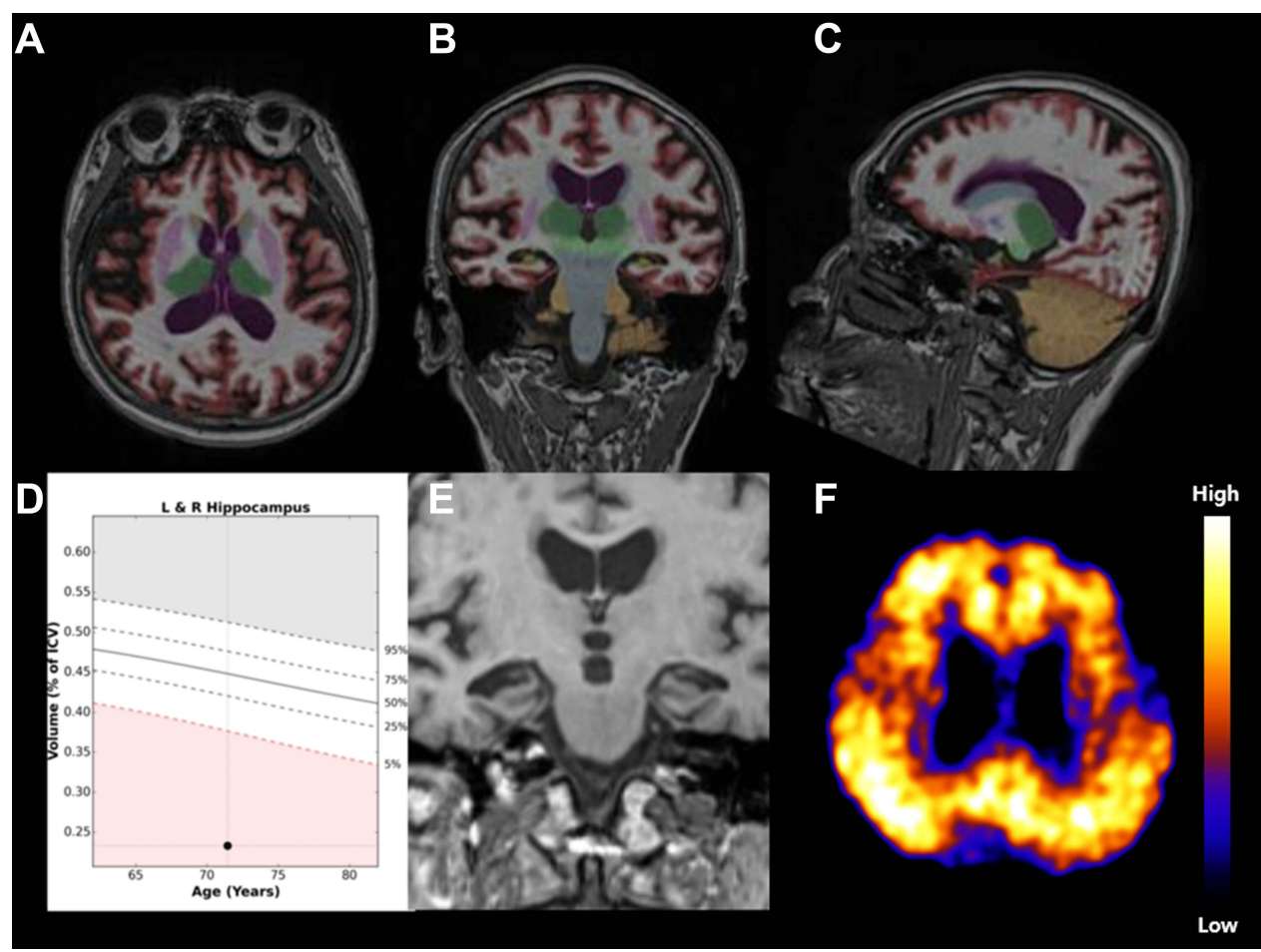

Figure 2 Representative 73-year-old woman with $\mathrm{MCl}$. (A-C): Output from analysis with NeuroQuant shows segmentation of regional brain volumes. (D): Age-matched reference charts provided by NeuroQuant showing \% of ICV and normative percentiles of hippocampal volume are 0.23 and I, respectively. The prediction score calculated from age and hippocampal \% of ICV was -9.44.(E): Coronal TI-weighted MR image shows hippocampal atrophy.(F): Positive amyloid imaging scans with "high" Pittsburgh compound $B$ retention.

Abbreviations: $\mathrm{MCl}$, mild cognitive impairment; L, left; R, right; ICV, intracranial volume (Courtesy of CorTechs Laboratories).
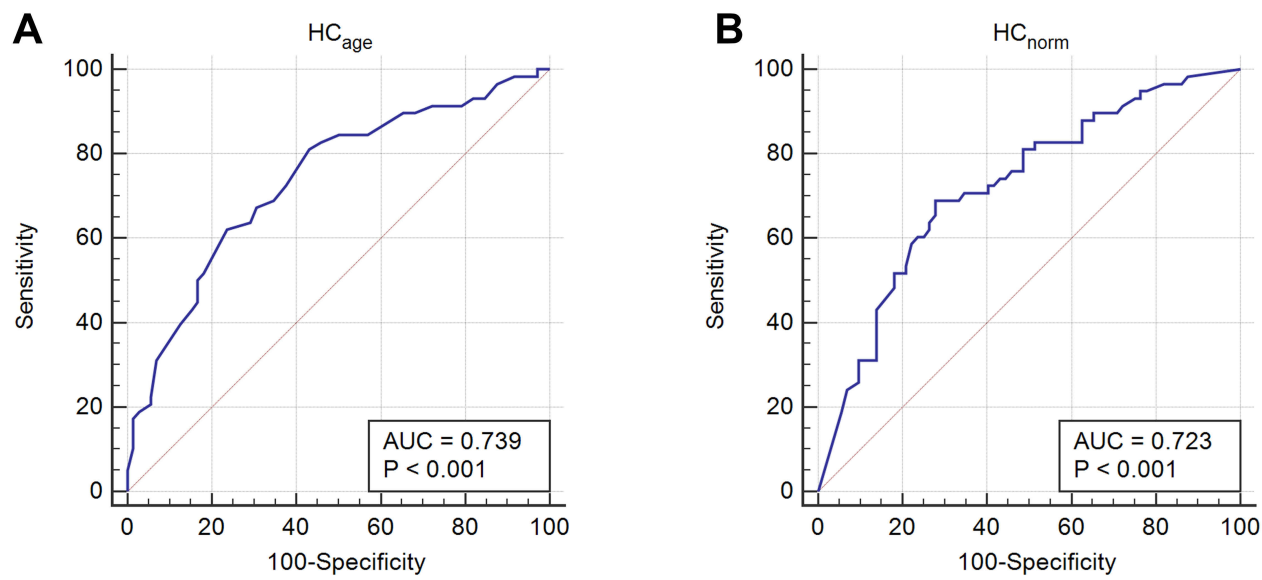

Figure $3 \mathrm{ROC}$ curves used to predict the presence of high amyloid deposition in patients with $\mathrm{MCl}$. (A) ROC curve for $\mathrm{HCage}$. (B) ROC curve for $\mathrm{HCnorm}$. Abbreviations: $\mathrm{MCl}$, mild cognitive impairment; $\mathrm{HCage}$, a prediction score calculated using age and the hippocampal volume percentage of intracranial volume; HCnorm, normative percentiles of hippocampal volume.

were not fully considered. ${ }^{40}$ In addition, we scanned one time for each participant and used only one MRI machine with the same protocol at a single center. Therefore, we could not investigate the intrascan or interscan variability, which may affect the volumetric measurement of the brain in real clinical settings. ${ }^{41,42}$

\section{Conclusion}

Our results suggest that relative hippocampal volume measures provided by NeuroQuant can be used to screen for cerebral $\mathrm{A} \beta$ positivity in amnestic MCI individuals in clinical practice. It may also help clinicians obtain additional information about MCI patients and determine early 
Table 4 Sensitivity, Specificity, Positive Predictive Value, Negative Predictive Value, and Accuracy at Various Cutoff Points of $\mathrm{HC}_{\text {age }}$ and $\mathrm{HC}_{\text {norm }}$ for Predicting $\mathrm{A} \beta$ Positivity in Patients with Mild Cognitive Impairment

\begin{tabular}{|c|c|c|c|c|c|}
\hline Cutoff & Sensitivity & Specificity & Positive Predictive Value & Negative Predictive Value & Accuracy \\
\hline \multicolumn{6}{|l|}{$H_{\text {age }}$} \\
\hline$>-14.6$ & 100 & 2.8 & 45.3 & 100.0 & 46.2 \\
\hline$>-14.1$ & 98.3 & 2.8 & 44.9 & 66.7 & 45.4 \\
\hline$>-13.6$ & 93.1 & 15.3 & 47.0 & 73.3 & 50.0 \\
\hline$>-13.1$ & 89.7 & 31.9 & 51.5 & 79.3 & 57.7 \\
\hline$>-12.6$ & 81.0 & 56.9 & 60.3 & 78.8 & 67.7 \\
\hline$>-12.1$ & 62.1 & 76.4 & 68.0 & 71.4 & 70.0 \\
\hline$>-11.6$ & 39.7 & 87.5 & 71.9 & 64.3 & 66.2 \\
\hline$>-11.1$ & 17.2 & 98.6 & 90.9 & 59.6 & 62.3 \\
\hline$>-10.6$ & 5.2 & 100.0 & 10.0 & 56.7 & 57.7 \\
\hline \multicolumn{6}{|l|}{$H_{\text {norm }}$} \\
\hline$\leq 91$ & 93.1 & 23.6 & 49.6 & 80.9 & 54.6 \\
\hline$\leq 82$ & 89.7 & 30.6 & 51.0 & 78.6 & 56.9 \\
\hline$\leq 73$ & 87.9 & 37.5 & 53.2 & 79.4 & 60.0 \\
\hline$\leq 64$ & 82.8 & 43.1 & 54.0 & 75.6 & 60.8 \\
\hline$\leq 55$ & 79.3 & 51.4 & 56.8 & 75.5 & 63.8 \\
\hline$\leq 46$ & 74.1 & 56.9 & 58.1 & 73.2 & 64.6 \\
\hline$\leq 37$ & 70.7 & 65.3 & 62.2 & 73.4 & 67.7 \\
\hline$\leq 28$ & 69.0 & 72.2 & 66.7 & 74.3 & 70.8 \\
\hline$\leq 19$ & 60.6 & 76.4 & 67.3 & 70.5 & 69.2 \\
\hline$\leq 10$ & 44.8 & 84.7 & 70.3 & 65.6 & 66.9 \\
\hline
\end{tabular}

Notes: The results of exploration on how the sensitivity, specificity, positive predictive value, negative predictive value and accuracy change as the $\mathrm{HC}_{\text {age }}$ increases from -14.6 to -10.6 by 0.5 . $Y=12.047+-0.087 X_{1}+-14.192 X_{2}$, where $Y$ is the prediction score of $\mathrm{HC}_{\text {age }}, X_{1}$ is age, and $X_{2}$ is \%HC/ICV.

Abbreviations: $\mathrm{A} \beta$, beta-amyloid; $\mathrm{HC}_{\text {norm }}$, normative percentiles of hippocampal volume.

active intervention for delaying the progression to $\mathrm{AD}$ dementia.

\section{Abbreviations}

$\% \mathrm{HC}$, hippocampal volume percentage; 3D, threedimensional; AD, Alzheimer's disease; aMCI, amnestic MCI; $\mathrm{A} \beta$, amyloid beta; $\mathrm{A} \beta+$, amyloid positive; $\mathrm{A} \beta$-, amyloid negative; CDR, clinical dementia rating; CERAD-K, Korean version of Consortium to Establish a Registry for Alzheimer's Disease; GM, gray matter; $\mathrm{HC}_{\mathrm{age}}$, prediction score calculated from age and $\% \mathrm{HC} / \mathrm{ICV} ; \mathrm{HC}_{\text {norm }}$, normative percentile of hippocampal volume; ICC, interclass coefficient; ICV, intracranial volume; KBASE, Korean brain aging study of early diagnosis and prediction of Alzheimer's disease; MCI, mild cognitive impairment; MRI, magnetic resonance imaging; NIA-AA, National Institute on Aging-Alzheimer's Association; NPV, negative predictive value; $\mathrm{PiB}$, Pittsburgh compound $\mathrm{B}$; $\mathrm{PET}$, positron emission tomography; PPV, positive predictive value; ROC, receiver operating characteristic; ROI, region of interest; SUVR, standardized uptake value ratio; WM, white matter; WMH, white matter hyperintensities.

\section{Data Sharing Statement}

The datasets used and/or analyzed during the present study are available from the corresponding author on reasonable request.

\section{Ethics Approval and Informed Consent}

This study protocol was approved by the Institutional Review Boards of Seoul National University Hospital and Seoul National University-Seoul Metropolitan Government Boramae Center, and all subjects provided written informed consent. The KBASE study is conducted in accordance with the Declaration of Helsinki.

\section{Acknowledgments}

The Medical Research Collaborating Center at Seoul National University Hospital kindly provided statistical advice for this manuscript.

\section{Author Contributions}

All authors contributed to data analysis, drafting or revising the article, gave final approval of the version to be published, and agree to be accountable for all aspects of the work. 


\section{Funding}

This study was supported by grants from the Ministry of Science and ICT (Grant No: NRF-2014M3C7A1046042 and 2018M3C7A1056888) and the SNUH research fund (Grant No: 04-20190500). The funders had no role in the design and conduct of the study; collection, management, analysis, and interpretation of the data; preparation, review, or approval of the manuscript; and decision to submit the manuscript for publication.

\section{Disclosure}

The authors report no conflicts of interest in this work.

\section{References}

1. Petersen RC, Smith GE, Waring SC, Ivnik RJ, Tangalos EG, Kokmen E. Mild cognitive impairment: clinical characterization and outcome. Arch Neurol. 1999;56(3):303-308. doi:10.1001/ archneur.56.3.303

2. Morris JC. Mild cognitive impairment is early-stage Alzheimer disease: time to revise diagnostic criteria. Arch Neurol. 2006;63 (1):15-16. doi:10.1001/archneur.63.1.15

3. Price JC, Klunk WE, Lopresti BJ, et al. Kinetic modeling of amyloid binding in humans using PET imaging and Pittsburgh Compound-B J Cereb Blood Flow Metab. 2005;25(11):1528-1547. doi:10.1038/sj. jcbfm. 9600146

4. Rowe $\mathrm{CC}, \mathrm{Ng} \mathrm{S}$, Ackermann U, et al. Imaging $\beta$-amyloid burden in aging and dementia. Neurology. 2007;68(20):1718-1725. doi:10.1212/01.wnl.0000261919.22630.ea

5. Wolk DA, Price JC, Saxton JA, et al. Amyloid imaging in mild cognitive impairment subtypes. Ann Neurol. 2009;65(5):557-568. doi:10.1002/ana. 21598

6. Nordberg A, Carter SF, Rinne J, et al. A European multicentre PET study of fibrillar amyloid in Alzheimer's disease. Eur $J$ Nucl Med Mol Imaging. 2013;40(1):104-114. doi:10.1007/s00259-012-2237-2

7. Jack Jr CR, Wiste HJ, Vemuri P, et al. Brain beta-amyloid measures and magnetic resonance imaging atrophy both predict time-toprogression from mild cognitive impairment to Alzheimer's disease. Brain. 2010;133(11):3336-3348. doi:10.1093/brain/awq277

8. McKhann GM, Knopman DS, Chertkow H, et al. The diagnosis of dementia due to Alzheimer's disease: recommendations from the National Institute on Aging-Alzheimer's Association workgroups on diagnostic guidelines for Alzheimer's disease. Alzheimers Dement. 2011;7(3):263-269. doi:10.1016/j.jalz.2011.03.005

9. Engelborghs S, Niemantsverdriet E, Struyfs H, et al. Consensus guidelines for lumbar puncture in patients with neurological diseases. Alzheimers Dement. 2017;8(1):111-126.

10. Lim YY, Maruff P, Getter C, Snyder PJ. Disclosure of positron emission tomography amyloid imaging results: a preliminary study of safety and tolerability. Alzheimers Dement. 2016;12(4):454-458. doi:10.1016/j.jalz.2015.09.005

11. Falgàs $\mathrm{N}$, Tort-Merino $\mathrm{A}$, Balasa $\mathrm{M}$, et al. Clinical applicability of diagnostic bio-markers in early-onset cognitive impairment. Eur J Neurol. 2019;26(8):1098-1104. doi:10.1111/ene.13945

12. Frisoni G, Fox N, Jack JC, Scheltens P, Thompson P. The clinical use of structural MRI in Alzheimer disease. Nat Rev Neurol. 2010;6 (2):67-77. doi:10.1038/nrneurol.2009.215

13. Tosun D, Schuff N, Mathis CA, Jagust W, Weiner MW; Initiative AsDN. Spatial patterns of brain amyloid- $\beta$ burden and atrophy rate associations in mild cognitive impairment. Brain. 2011;134 (4):1077-1088. doi:10.1093/brain/awr044
14. Jenkinson M, Beckmann CF, Behrens TE, Woolrich MW, Smith SM. Fsl. Neuroimage. 2012;62(2):782-790. doi:10.1016/j.neuroimage.201 1.09.015

15. Fischl B. FreeSurfer. Neuroimage. 2012;62(2):774-781. doi:10.1016/ j.neuroimage.2012.01.021

16. Ashburner J, Friston KJ. Unified segmentation. Neuroimage. 2005;26 (3):839-851. doi:10.1016/j.neuroimage.2005.02.018

17. Brewer J, Magda S, Airriess C, Smith M. Fully-automated quantification of regional brain volumes for improved detection of focal atrophy in Alzheimer disease. Am $J$ Neuroradiol. 2009;30 (3):578-580. doi:10.3174/ajnr.A1402

18. Persson K, Selbæk G, Brækhus A, Beyer M, Barca M, Engedal K. Fully automated structural MRI of the brain in clinical dementia workup. Acta Radiol. 2017;58(6):740-747. doi:10.1177/02841851 16669874

19. Tanpitukpongse T, Mazurowski M, Ikhena J, Petrella J; Initiative AsDN. Predictive utility of marketed volumetric software tools in subjects at risk for Alzheimer disease: do regions outside the hippocampus matter? Am J Neuroradiol. 2017;38(3):546-552. doi:10.31 74/ajnr.A5061

20. Byun MS, Yi D, Lee JH, et al. Korean brain aging study for the early diagnosis and prediction of Alzheimer's disease: methodology and baseline sample characteristics. Psychiatry Investig. 2017;14 (6):851-863. doi:10.4306/pi.2017.14.6.851

21. Albert MS, DeKosky ST, Dickson D, et al. The diagnosis of mild cognitive impairment due to Alzheimer's disease: recommendations from the National Institute on Aging-Alzheimer's Association workgroups on diagnostic guidelines for Alzheimer's disease. Alzheimers Dement. 2011;7(3):270-279. doi:10.1016/j.jalz.2011.03.008

22. Ross DE, Ochs AL, Seabaugh JM, Shrader CR. Man versus machine: comparison of radiologists' interpretations and neuroquant ${ }^{\circledR}$ volumetric analyses of brain MRIs in patients with traumatic brain injury. J Neuropsychiatry Clin Neurosci. 2013;25(1):32-39. doi:10.1176/ appi.neuropsych. 11120377

23. Reiman EM, Chen K, Liu X, et al. Fibrillar amyloid- $\beta$ burden in cognitively normal people at 3 levels of genetic risk for Alzheimer's disease. Proc Natl Acad Sci. 2009;106(16):6820-6825. doi:10.1073/ pnas.0900345106

24. Tzourio-Mazoyer N, Landeau B, Papathanassiou D, et al. Automated anatomical labeling of activations in SPM using a macroscopic anatomical parcellation of the MNI MRI single-subject brain. Neuroimage. 2002;15(1):273-289. doi:10.1006/nimg.2001.0978

25. Lopresti BJ, Klunk WE, Mathis CA, et al. Simplified quantification of Pittsburgh Compound B amyloid imaging PET studies: a comparative analysis. J Nucl Med. 2005;46(12):1959-1972.

26. Jack CR, Wiste HJ, Weigand SD, et al. Amyloid-first and neurodegeneration-first profiles characterize incident amyloid PET positivity. Neurology. 2013;81(20):1732-1740. doi:10.1212/01. wnl.0000435556.21319.e4

27. Ochs AL, Ross DE, Zannoni MD, Abildskov TJ, Bigler ED. Comparison of automated brain volume measures obtained with NeuroQuant ${ }^{\circledR}$ and FreeSurfer. $J$ Neuroimaging. 2015;25(5):721-727. doi:10.1111/jon.12229

28. Ross DE, Ochs AL, DeSmit ME, Seabaugh JM, Havranek MD. Alzheimer's disease neuroimaging I. man versus machine part 2: comparison of radiologists' interpretations and neuroquant measures of brain asymmetry and progressive atrophy in patients with traumatic brain injury. J Neuropsychiatry Clin Neurosci. 2015;27 (2):147-152. doi:10.1176/appi.neuropsych. 13040088

29. Ross DE, Ochs AL, Seabaugh J, Henshaw T. NeuroQuant ${ }^{\circledR}$ revealed hippocampal atrophy in a patient with traumatic brain injury. J Neuropsychiatr Clin Neurosci. 2012;24(1):E33-E33. doi:10.1176/ appi.neuropsych.11020044

30. Farid N, Girard HM, Kemmotsu N, et al. Temporal lobe epilepsy: quantitative MR volumetry in detection of hippocampal atrophy Radiology. 2012;264(2):542-550. doi:10.1148/radiol.12112638 
31. Azab M, Carone M, Ying SH, Yousem DM. Mesial temporal sclerosis: accuracy of NeuroQuant versus neuroradiologist. Am $J$ Neuroradiol. 2015;36(8):1400-1406. doi:10.3174/ajnr.A4313

32. Kovacevic S, Rafii MS, Brewer JB. High-throughput, fully-automated volumetry for prediction of MMSE and CDR decline in mild cognitive impairment. Alzheimer Dis Assoc Disord. 2009;23 (2):139. doi:10.1097/WAD.0b013e318192e745

33. Heister D, Brewer JB, Magda S, Blennow K, McEvoy LK; Initiative AsDN. Predicting MCI outcome with clinically available MRI and CSF biomarkers. Neurology. 2011;77(17):1619-1628. doi:10.1212/ WNL.0b013e3182343314

34. Fischl B, Salat DH, Busa E, et al. Whole brain segmentation: automated labeling of neuroanatomical structures in the human brain. Neuron. 2002;33(3):341-355. doi:10.1016/S0896-6273(02)00569-X

35. Sperling RA, Aisen PS, Beckett LA, et al. Toward defining the preclinical stages of Alzheimer's disease: recommendations from the National Institute on Aging-Alzheimer's Association workgroups on diagnostic guidelines for Alzheimer's disease. Alzheimers Dement. 2011;7(3):280-292. doi:10.1016/j.jalz.2011.03.003

36. Jack Jr CR, Knopman DS, Jagust WJ, et al. Hypothetical model of dynamic biomarkers of the Alzheimer's pathological cascade. Lancet Neurol. 2010;9(1):119-128. doi:10.1016/S1474-4422(09)70299-6

37. Frisoni GB, Fox NC, Jack Jr CR, Scheltens P, Thompson PM. The clinical use of structural MRI in Alzheimer disease. Nat Rev Neurol. 2010;6(2):67.
38. Fjell AM, McEvoy L, Holland D, Dale AM, Walhovd KB; Initiative AsDN. What is normal in normal aging? Effects of aging, amyloid and Alzheimer's disease on the cerebral cortex and the hippocampus. Prog Neurobiol. 2014;117:20-40. doi:10.1016/j.pneurobio.2014.02. 004

39. Jack Jr CR. Alzheimer disease: new concepts on its neurobiology and the clinical role imaging will play. Radiology. 2012;263(2):344-361. doi:10.1148/radiol.12110433

40. Zivadinov R, Jakimovski D, Gandhi S, et al. Clinical relevance of brain atrophy assessment in multiple sclerosis. Implications for its use in a clinical routine. Expert Rev Neurother. 2016;16:777-793. doi:10.1080/14737175.2016.1181543

41. Jovicich J, Czanner S, Han X, et al. MRI-derived measurements of human subcortical, ventricular and intracranial brain volumes: reliability effects of scan sessions, acquisition sequences, data analyses, scanner upgrade, scanner vendors and field strengths. Neuroimage. 2009;46(1):177-192. doi:10.1016/j.neuroimage.2009.02.010

42. Ewers M, Teipel SJ, Dietrich O, et al. Multicenter assessment of reliability of cranial MRI. Neurobiol Aging. 2006;27(8):1051-1059. doi:10.1016/j.neurobiolaging.2005.05.032

\section{Publish your work in this journal}

Neuropsychiatric Disease and Treatment is an international, peerreviewed journal of clinical therapeutics and pharmacology focusing on concise rapid reporting of clinical or pre-clinical studies on a range of neuropsychiatric and neurological disorders. This journal is indexed on PubMed Central, the 'PsycINFO' database and CAS, and is the official journal of The International Neuropsychiatric Association (INA). The manuscript management system is completely online and includes a very quick and fair peer-review system, which is all easy to use. Visit http://www.dovepress.com/testimonials.php to read real quotes from published authors. 